\title{
MicroRNA-101 regulates autophagy, proliferation and apoptosis via targeting EZH2 in laryngeal squamous cell carcinoma
}

\author{
L. CHEN ${ }^{1}$, J. JIA ${ }^{2}$, Y. ZANG ${ }^{1}$, J. LI ${ }^{1}$, B. WAN ${ }^{1, *}$ \\ ${ }^{1}$ Department of Otolaryngology, Henan Provincial People's Hospital, People's Hospital of Zhengzhou University, Zhengzhou, 450003, China; \\ ${ }^{2}$ Department of Otolaryngology, People's Hospital of Xixian, Xinyang, 464300, China \\ *Correspondence: wanbaoluo2016@163.com
}

Received August 11, 2018 / Accepted December 19, 2018

\begin{abstract}
Laryngeal carcinoma is the second commonest head and neck carcinoma globally. MicroRNA-101 (miR-101) has been reported as a tumor suppressor in multiple malignancies including laryngeal squamous cell carcinoma (LSCC). However, the roles and molecular mechanisms of miR-101 in the development of LSCC have not been fully elucidated. In the present study, RT-qPCR assay was performed to detect the expression of miR-101 and enhancer of zeste homolog 2 (EZH2) mRNA. Western blot assay was conducted to determine protein expression of LC3-I, LC3-II, p62 and EZH2. Cell proliferative capacity was evaluated by MTS assay. The effect of miR-101 alone or along with EZH2 on cell apoptosis was assessed by apoptotic index and caspase-3 activity. Bioinformatic analysis, luciferase assay and RNA immunoprecipitation (RIP) assay were carried out to investigate the interaction between miR-101 and EZH2. Results revealed that miR-101 expression was strikingly down-regulated in LSCC cell lines. Functional analyses showed that ectopic expression of miR-101 suppressed cell autophagy and proliferation and facilitated cell apoptosis in LSCC. Further investigations revealed that miR-101 inhibited EZH2 expression by direct interaction and EZH2 was highly expressed in LSCC cells. Also, EZH2 knockdown reduced the autophagic activity of LSCC cells. Moreover, restoration experiments showed that EZH2 up-regulation weakened miR-101mediated anti-autophagy, anti-proliferation and pro-apoptosis effects in LSCC cells. In conclusion, our findings suggested that miR-101 inhibited autophagy and proliferation and promoted apoptosis via targeting EZH2 in LSCC, providing a deep insight into the pathogenesis of LSCC and hinting the pivotal roles of epigenetic modifications especially histone methylation in the development of LSCC.
\end{abstract}

Key words: laryngeal squamous cell carcinoma, microRNA-101, EZH2, autophagy, proliferation, apoptosis

Laryngeal carcinoma is the second commonest head and neck carcinoma worldwide $[1,2]$, with an estimated 13150 new cases and 3710 deaths in the USA alone in 2018 [3]. Laryngeal squamous cell carcinoma (LSCC), the most frequent type of laryngeal carcinoma, has a high recurrent rate and low disease-free survival rate $[4,5]$. Although great advances have been achieved in the management of LSCC, the clinical outcomes for LSCC remain unsatisfied $[2,4,6]$.

MicroRNAs (miRNAs), a class of short endogenous non-coding transcripts with the length about 22 nucleotides, can regulate gene expression at post-transcriptional levels [7]. Accumulating evidence shows that miRNAs can control a wide range of carcinoma-related cellular processes such as proliferation, apoptosis and autophagy to induce or hinder tumorigenesis and progression [8-10]. Moreover, some studies pointed out that the dysregulation of miRNAs and epigenetic pathways was closely linked with the pathogenesis of carcinomas [11, 12]. MicroRNA-101 (miR-101) has been reported as a tumor suppressor in multiple carcinomas such as gastric carcinoma [13], endometrial carcinoma [14] and breast carcinoma [15]. For instance, ectopic expression of miR-101 suppressed proliferation and invasion and induced apoptosis by down-regulating cyclooxygenase- 2 in cervical carcinoma cells [16]. MiR-101 inhibited proliferation and promoted apoptosis by targeting DNA methyltransferase 3A in lung carcinoma [17]. Additionally, Li et al. revealed that exogenous expression of miR-101 induced the reduction of cell proliferative and migratory capacities and the increase of cell apoptotic rate in LSCC [18]. However, the roles and molecular mechanisms of miR-101 in the development and progression of LSCC need to be further explored.

In the present study, we demonstrated that miR-101 suppressed cell autophagy and proliferation and promoted cell apoptosis by targeting enhancer of zeste homolog 2 
(EZH2) in LSCC, deepening our understanding on etiology of LSCC.

\section{Materials and methods}

Cell culture. Human LSCC cell lines Hep-2, AMC-HN-8 and TU-177 were purchased from the American Type Culture Collection (ATCC, Manassas, VA, USA). Human LSCC cell line TU-212 was obtained from Cell Bank of the Chinese Academy of Sciences (Shanghai, China). LSCC cell lines (TU-177, AMC-HN-8, Hep-2, TU-212) were maintained in RPMI-1640 medium (Gibco, Carlsbad, CA, USA) containing 10\% FBS (Gibco) and $1 \%$ penicillin/streptomycin (Invitrogen, Carlsbad, CA, USA). Normal human oral keratinocytes (NHOKs) were ordered from the Institute of Biochemistry and Cell Biology of the Chinese Academy of Sciences (Shanghai, China) and cultured in defined keratinocyte-serum free medium (KSFM, Thermo Scientific, Waltham, MA, USA).

Reagents and cell transfection. MiR-101 mimic and its negative control (miR-NC), miR-101 inhibitor (anti-miR101 ) and its negative control (anti-miR-NC) were purchased from GenePharma Co., Ltd (Shanghai, China). The fulllength fragments of EZH2 coding region were inserted into pcDNA3.1 vector (Thermo Scientific) to produce pcDNA3.1-EZH2 (EZH2) overexpression plasmid. All transfection procedures were performed using Lipofectamine 2000 reagent (Thermo Scientific) following the instructions of manufacturer.

Reverse transcription-quantitative PCR (RT-qPCR) assay. Total RNAs were isolated from LSCC cells using TRIzol reagent (Thermo Scientific) referring to the protocols of manufacturer. For the expression analysis of EZH2, RNAs were reversely transcribed into cDNAs by M-MLV Reverse Transcriptase (Thermo Scientific) and random primers. Then, real time-quantitative PCR analysis was conducted using SYBR Select Master Mix (Thermo Scientific) and specific primers. GAPDH functions as the house-keeping gene to normalize the expression of EZH2. Quantitative primers for EZH2 and GAPDH were presented as follows: EZH2, 5'-CCCTGACCTCTGTCTTACTTGTGGA-3' (forward) and 5'-ACGTCAGATGGTGCCAGCAATA-3' (reverse); GAPDH, 5'-GAAGGTGAAGGTCGGAGTC-3' (forward) and 5'-GAAGATGGTGATGGGATTTC-3' (reverse). For expression analysis of miR-101, cDNAs were synthesized by TaqMan ${ }^{m}$ Advanced miRNA cDNA Synthesis Kit (Thermo Scientific). Then, quantitative analysis of miR-101 was performed using TaqMan Advanced miRNA Assay reagents and primers (Thermo Scientific). The relative level of miR-101 was normalized to that of U6 snRNA. Data analysis was performed by $2^{-\Delta \Delta C t}$ method.

Western blot assay. LSCC cells were collected and lysed by RIPA Lysis and Extraction Buffer (Thermo Scientific) supplemented with protease inhibitor cocktail (Thermo Scientific). After high-speed centrifugation, proteins in the supernatants were quantified through Pierce BCA Protein Assay Kit (Thermo Scientific). Then, equal amounts of proteins were separated through SDS-PAGE and blotted on PDVF membranes (Millipore, Bedford, MA, USA). After the blockade of non-specific protein signals, the membranes were probed overnight at $4{ }^{\circ} \mathrm{C}$ with anti-LC3 (LC3-I and LC3-II) antibody (1:1000 dilution, 14600-1-AP, Proteintech Inc., Rosemont, IL, USA), anti-SQSTM1/p62 antibody (1:2000 dilution, ab56416, Abcam, Cambridge, UK), anti-EZH2 antibody (1:1000 dilution, ab228697, Abcam), and anti- $\beta$-actin antibody (1:2000 dilution, ab8227, Abcam). Next, the membranes were incubated for $1.5 \mathrm{~h}$ at room temperature with Goat Anti-Rabbit IgG H\&L (HRP) (1:5000 dilution, ab6721, Abcam) or Goat Anti-Mouse IgG H\&L (HRP) (1:5000 dilution, ab6789, Abcam) secondary antibody. Finally, protein bands were visualized by Pierce ${ }^{\text {Tm }}$ ECL Western Blotting Substrate (Thermo Scientific) on Bio-Rad ChemiDoc MP imaging system (Bio-Rad Laboratories, Hercules, CA, USA) and were quantified by Bio-Rad Image Lab software.

RNA immunoprecipitation (RIP) assay. Hep- 2 cells were transfected with miR-NC for miR-101 mimic. At $48 \mathrm{~h}$ after transfection, RIP assay was conducted through Magna RIP ${ }^{\mathrm{m}}$ RNA-Binding Protein Immunoprecipitation Kit (Millipore) referring to the protocols of manufacturer. Briefly, transfected cells were collected and lysed using RIP Lysis Buffer. Then, cell lysate was precleared with protein $\mathrm{A} / \mathrm{G}$ magnetic beads for $1 \mathrm{~h}$ at $4^{\circ} \mathrm{C}$. Next, precleared lysate was incubated overnight at $4{ }^{\circ} \mathrm{C}$ with protein $\mathrm{A} / \mathrm{G}$ magnetic beads and antiArgonaute2 (Ago2, Millipore) or anti-IgG antibody (Millipore). After washing, proteins in the IgG or Ago2 immunoprecipitation complex were removed with proteinase $\mathrm{K}$ buffer and RNAs were extracted according to the instructions of manufacturer. Finally, the enrichment degrees of EZH2 in IgG or Ago2 immunoprecipitation complex were determined by RT-qPCR assay.

MTS assay. Cell proliferative ability was measured at the indicated time points $(0,24,48,72 \mathrm{~h})$ after transfection using a MTS Assay Kit (Colorimetric) (Abcam) according to the protocols of manufacturer. Briefly, cells were seeded into 96 -well microtiter plates at a final volume of $200 \mu \mathrm{l} /$ well. Then, cells were transfected with corresponding oligonucleotides or plasmids. At $0 \mathrm{~h}, 24 \mathrm{~h}, 48 \mathrm{~h}, 72 \mathrm{~h}$ post transfection, $20 \mu \mathrm{l}$ of MTS reagent was added into each well. After incubation for $4 \mathrm{~h}$ at $37^{\circ} \mathrm{C}$, cell absorbance was determined at the wavelength of $490 \mathrm{~nm}$.

Caspase-3 activity determination. Caspase-3 activity was measured at $48 \mathrm{~h}$ upon transfection using a Caspase 3 Assay Kit (Colorimetric) (Abcam) following the instructions of manufacturer. Briefly, cells were lysed using cell lysis buffer and protein concentration was determined using a Pierce BCA Protein Assay Kit (Thermo Scientific). Then, $50 \mu$ of $2 \times$ Reaction Buffer (containing $10 \mathrm{mM}$ DTT) was added into each protein sample $(50-200 \mu \mathrm{g} / 50 \mu \mathrm{l}$ cell lysis buffer). Next, the samples were incubated with $5 \mu \mathrm{l}$ of DEVD-p-NA 
substrate $(4 \mathrm{mM})$ for $2 \mathrm{~h}$ at $37^{\circ} \mathrm{C}$. At last, optical density values were measured at the wavelength of $400 \mathrm{~nm}$.

Apoptotic index detection. Cell apoptotic index was detected at $48 \mathrm{~h}$ post transfection using an Annexin V-FITC Apoptosis Detection Kit (Sigma-Aldrich, St. Louis, MO, USA) referring to the protocols of manufacturer. Generally, cells were resuspended in $1 \times$ Binding Buffer $(500 \mu \mathrm{l})$ at a concentration of $\sim 1 \times 10^{6}$ cells $/ \mathrm{ml}$ and then incubated with $5 \mu \mathrm{l}$ of Annexin V-FITC conjugate and $10 \mu \mathrm{l}$ of propidium iodide solution for $10 \mathrm{~min}$ in the dark. Finally, cell apoptotic index was determined using a flow cytometry (BD Biosciences, San Jose, CA, USA).

Luciferase reporter assay. Partial sequences of EZH2 3'UTR containing predicted miR-101 binding sites were inserted into psiCHECK-2 luciferase vector (Promega, Madison, WI, USA) to yield EZH2-WT-3'UTR reporter. Also, KOD-plus-mutagenesis kit (Toyobo, Osaka, Japan) was employed to generate EZH2-MUT-3'UTR reporter with mutant miR-101 binding sites. Then, EZH2-WT-3'UTR or EZH2-MUT-3'UTR reporter was transfected into HEK293T cells along with or without miR-101 mimic. At $48 \mathrm{~h}$ after transfection, luciferase activities were determined through a dual luciferase reporter assay (Promega).

Statistical analysis. Data were shown as mean \pm standard deviation from more than 3 independent experiments.
Difference analysis between two group data was carried out by Student's t-test with $\mathrm{p}<0.05$ as statistically significant. Data was analyzed by GraphPad software (Version 3.0; GraphPad, San Diego, CA, USA).

\section{Results}

MiR-101 was lower expressed in LSCC cell lines and miR-101 overexpression inhibited autophagy in LSCC cells. Firstly, RT-qPCR assay revealed that miR-101 expression was markedly down-regulated in four types of LSCC cell lines (TU-177, AMC-HN-8, Hep-2, TU-212) than that in NHOK cells (Figure 1A), indicating that miR-101 was implicated in the pathogenesis of LSCC. To further validate this conjecture, miR-101 mimic and its scramble control (miR-NC) were synthesized and then transfected into Hep-2 and TU-212 cells, respectively. As presented in Figure 1B, the transfection of miR-101 mimic induced a striking up-regulation of miR-101 level in Hep-2 and TU-212 cells, denoting that miR-101 mimic could be used for the subsequent gainof-function experiments. Further analyses revealed that the up-regulation of miR-101 resulted in a notable reduction of LC3-II/LC3-I ratio and conspicuous increase of p62 protein expression in Hep-2 and TU-212 cells (Figure 1C), signifying that miR-101 suppressed autophagy in LSCC cells.
A

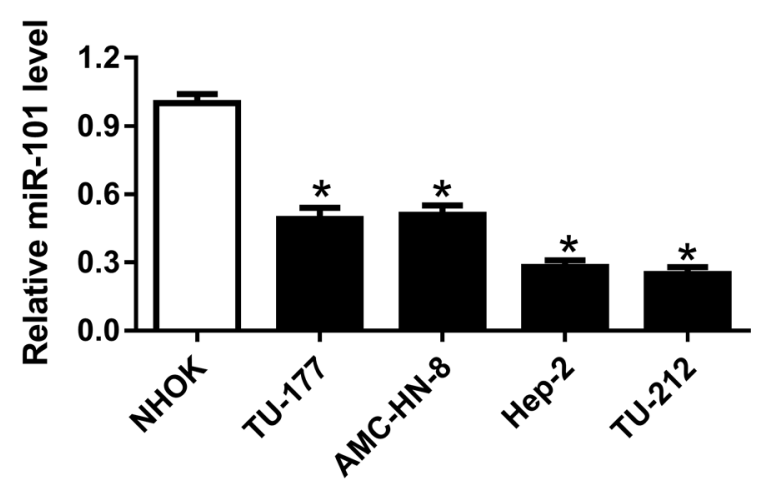

B

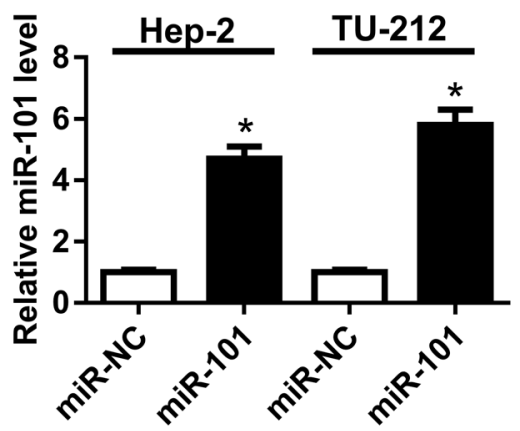

C
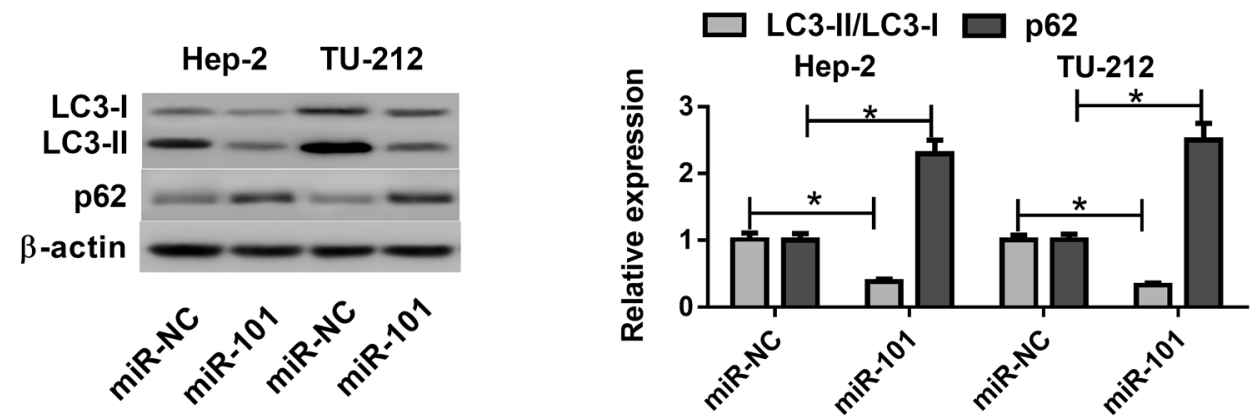

Figure 1. MiR-101 was lower expressed in LSCC cell lines and miR-101 overexpression inhibited autophagy in LSCC cells. A) MiR-101 level was measured by RT-qPCR assay in LSCC cell lines (TU-177, AMC-HN-8, Hep-2, TU-212) and NHOK cells. B) Hep-2 and TU-212 cells were transfected with miR-101 mimic or miR-NC. Then, miR-101 level was detected by RT-qPCR assay at $48 \mathrm{~h}$ after transfection, C) LC3-II/LC3-I ratio and p62 protein level were determined by western blot assay at $48 \mathrm{~h}$ post transfection. ${ }^{*} \mathrm{p}<0.05$. 
Enforced expression of miR-101 inhibited proliferation and induced apoptosis in LSCC cells. Then, MTS assay showed that enforced expression of miR-101 curbed the proliferation of Hep-2 and TU-212 cells (Figure 2A and 2B). Moreover, flow cytometry analyses disclosed that miR-101 overexpression resulted in an obvious elevation of apoptosis index in Hep-2 and TU-212 cells (Figures 2C and 2D). Also, increased caspase- 3 activity was observed in Hep-2 and TU-212 cells transfected with miR-101 mimic as compared to that in miR-NC-transfected cells (Figures $2 \mathrm{E}$ and $2 \mathrm{~F}$ ). In a word, these results manifested that miR-101 overexpression suppressed proliferation and induced apoptosis in LSCC cells.

EZH2 was a downstream target of miR-101. Next, TargetScan online website was used to predict possible targets of miR-101. Among candidate targets of miR-101, EZH2 was chosen because of its potential oncogenic roles in LSCC [19-21] (Figure 3A). To further confirm this predic-

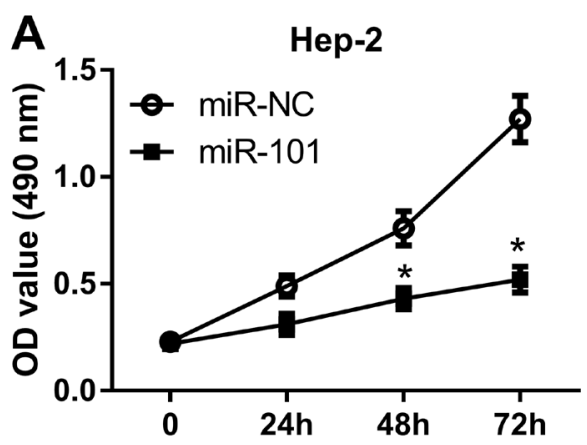

B

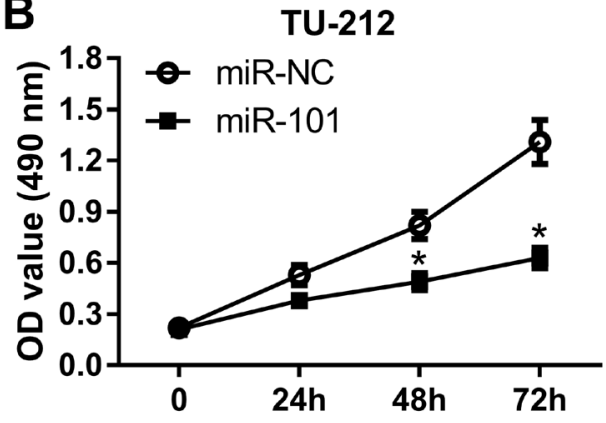

C

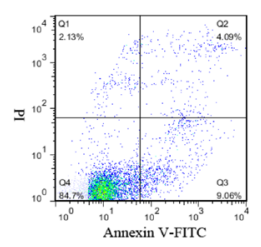

Hep-2
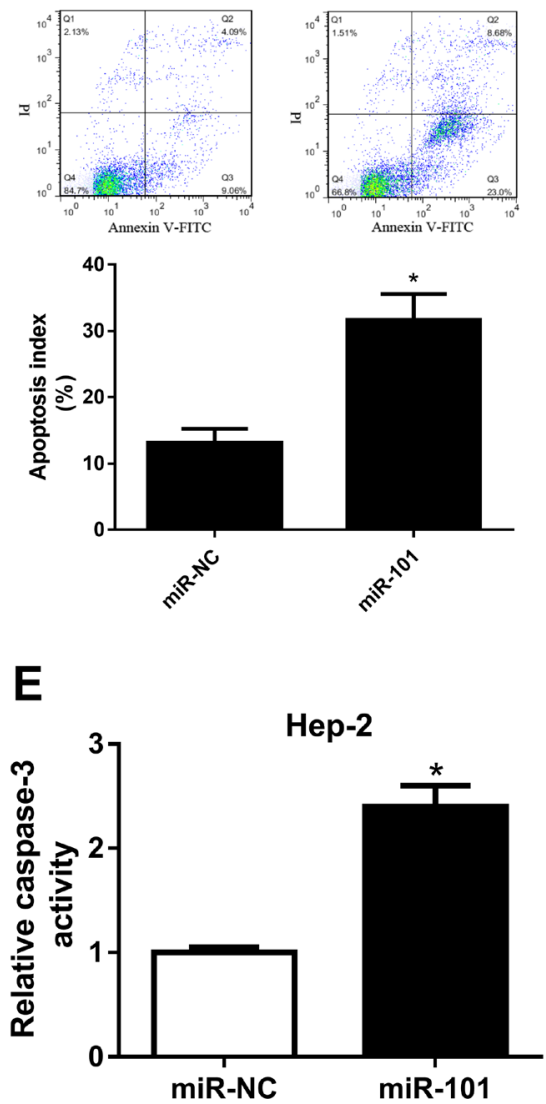

D

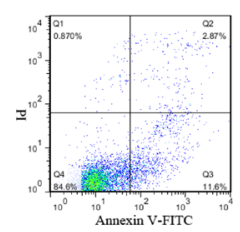

TU-212

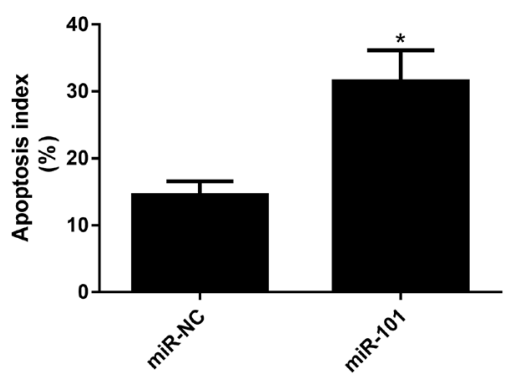

$\mathbf{F}$

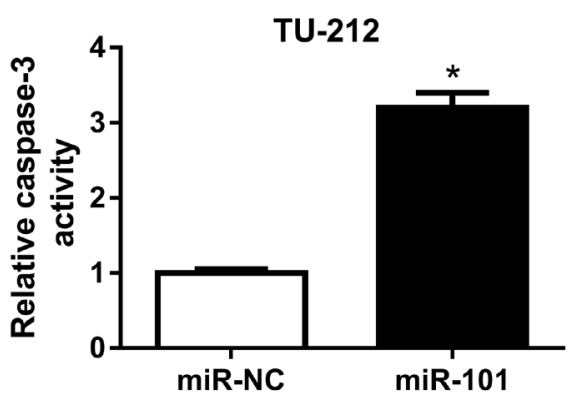

Figure 2. Enforced expression of miR-101 inhibited proliferation and induced apoptosis in LSCC cells. Hep-2 and TU-212 cells were transfected with miR-NC or miR-101. Then, cell proliferation capacity was assessed by MTS assay at $0,24 \mathrm{~h}, 48 \mathrm{~h}$, or $72 \mathrm{~h}$ upon transfection (A and B), apoptosis index $(\mathrm{C}$ and $\mathrm{D})$ and caspase-3 activity ( $\mathrm{E}$ and $\mathrm{F}$ ) were determined at $48 \mathrm{~h}$ following transfection. ${ }^{*} \mathrm{p}<0.05$. 
A

\section{EZH2-WT 5'...CUUCAGGAACCUCGAGUACUGUG...3'<smiles>C1#CC#C1</smiles> \\ miR-101 3' AAGUCAAUAGUGUCAUGACAU 5' \\ EZH2-MT 5'...CUUCAGGAACCUCGAAGCAGUCG...3' \\ miR-101 3' AAGUCAAUAGUGUCAUGACAU 5'}

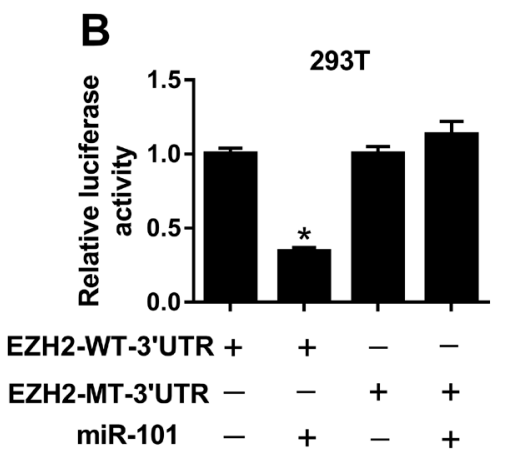

C
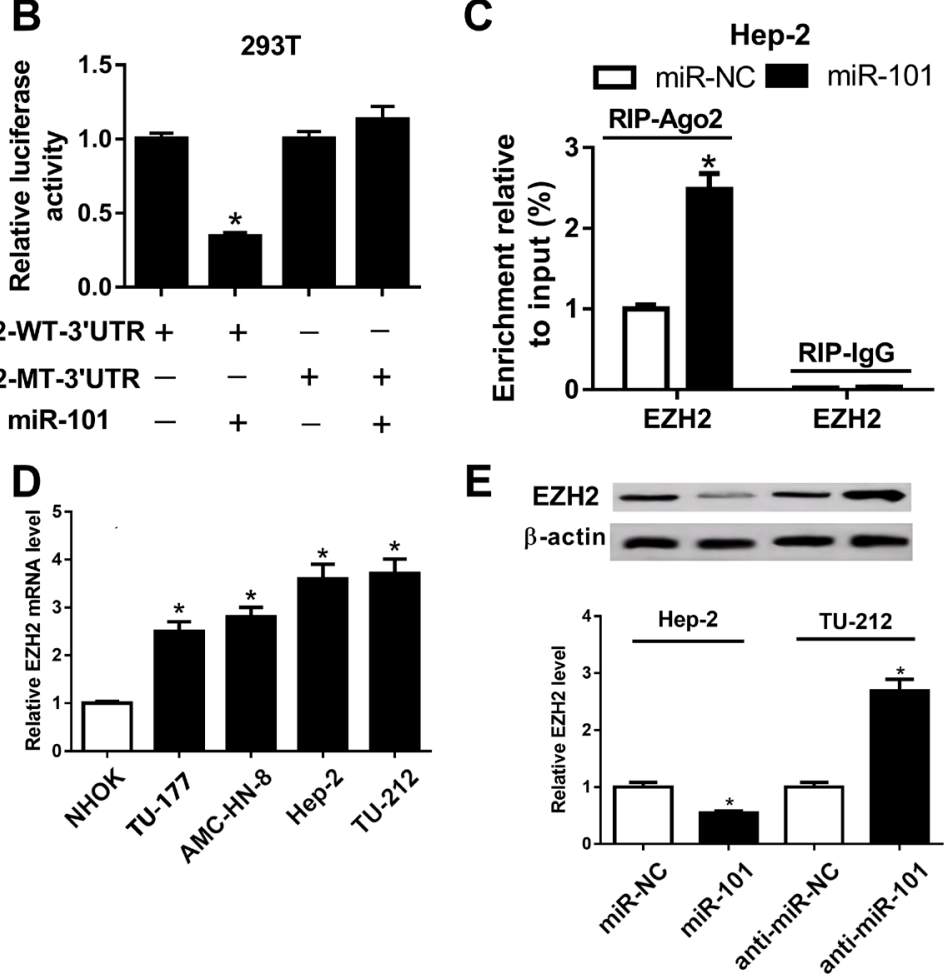

Figure 3. EZH2 was a downstream target of miR-101. A) Predicted binding sites between miR-101 and EZH2 3'UTR, and mutant sites in EZH2-MUT3'UTR reporter. B) The effect of miR-101 on luciferase activities of EZH2-WT-3'UTR or EZH2-MUT-3'UTR reporter was determined at 48 h upon transfection in HEK 293T cells. C) Hep-2 cells were transfected with miR-NC or miR-101 mimic. At 48 h after transfection, RIP and RT-qPCR assays were performed to determine the enrichment degree of EZH2 in IgG or Ago2 immunoprecipitation complex. D) RT-qPCR assay was conducted to measure miR-101 level in NHOK, TU-177, AMC-HN-8, Hep-2, and TU-212 cells. E) Hep-2 cells were transfected with miR-NC or miR-101 mimic, and TU212 cells were transfected with anti-miR-NC or anti-miR-101. At $48 \mathrm{~h}$ post transfection, EZH 2 protein level was detected by western blot assay. ${ }^{\star}$ p $<0.05$.

tion, EZH2-WT-3'UTR reporter with putative miR-101 binding sites and EZH2-MUT-3'UTR reporter containing mutant miR-101 binding sites were constructed, respectively. Then, the effect of miR-101 on luciferase activities of EZH2-WT-3'UTR or EZH2-MUT-3'UTR reporter was examined. Results showed that the introduction of miR-101 mimic markedly reduced the luciferase activity of EZH2-WT-3'UTR reporter in HEK293T cells but had no impact on the luciferase activity of EZH2-MUT-3'UTR reporter (Figure 3B), implying that miR-101 could interact with EZH2 3'UTR by putative binding sites. Ago2, a core component of RNA-induced silencing complex (RISC), plays pivotal roles in miRNA processing and gene silencing [22]. To further explore the interaction among miR-101,
RISC and EZH2, RIP assay was performed using IgG or Ago2 antibody in Hep-2 cells transfected with miR-NC or miR-101 mimic. Results showed that miR-101 overexpression resulted in the copious enrichment of EZH2 in Ago2 immunoprecipitation complex (Figure 3C), further indicating that miR-101 could bind with EZH2. Next, RT-qPCR assay further showed that EZH2 expression was strikingly up-regulated in four types of LSCC cell lines as compared to that in NHOK cells (Figure 3D). Moreover, EZH2 expression was notably reduced in miR-101-overexpressed Hep-2 cells but was remarkably increased in miR-101-deficient TU-212 cells (Figure 3E). Taken together, these results showed that miR-101 inhibited target EZH2 expression by direct interaction. 
EZH2 up-regulation abrogated the inhibitory effect of miR-101 on autophagy in LSCC cells. Next, our outcomes disclosed that EZH2 knockdown could abate the autophagic activity of LSCC cells, as evidenced by reduced LC3-II/ LC3-I ratio and increased p62 expression in si-EZH2-transfected Hep-2 and TU-212 cells (Figure 4). Further analyses revealed that the restoration of EZH2 expression resulted in the increase of LC3-II/LC3-I ratio and the reduction of p62 protein level in miR-101 overexpressed Hep-2 and TU-212 cells. That was to say, EZH2 up-regulation abrogated the inhibitory effect of miR-101 on autophagy in LSCC cells.

$\mathrm{EZH} 2$ restoration weakened $\mathrm{miR}-101-$ mediated antiproliferation and pro-apoptosis effects in LSCC cells. Next, MTS assay revealed that the inhibitory effect of miR-101 on cell proliferation was markedly relieved by increased EZH2 in Hep-2 and TU-212 cells (Figures 5A and 5B). Also, up-regulated EZH2 strikingly inhibited miR-101-induced apoptosis in Hep-2 and TU-212 cells, as presented by the reduction of apoptosis index (Figures 5C and 5D) and caspase- 3 activity (Figures $5 \mathrm{E}$ and $5 \mathrm{~F}$ ) in miR-101-transfected cells upon the overexpression of EZH2.

\section{Discussion}

LSCC is a serious threat for human health and life, giving rise to an enormous financial burden for individual, family and society $[3,23]$. Mounting miRNAs have been reported to be abnormally expressed in LSCC [24]. Also, prior studies showed that some miRNAs were implicated in the pathogenesis of LSCC. For example, ectopic expression of microRNA-195 resulted in the notable reduction of cell proliferative, migratory and invasive abilities by targeting ROCK1 in LSCC [25]. MicroRNA-204-5p hampered cell proliferation, migration and invasion by targeting forkhead box C1 in LSCC [26].

As mentioned above, miR-101 has been widely reported as a tumor suppressor in a variety of malignancies including LSCC. Also, some studies indicated that miR-101 could function as a potential indicator of better prognosis in multiple carcinomas $[27,28]$. In the present study, we aimed to further investigate the roles and molecular mechanisms of miR-101 in the LSCC development. Our results disclosed that miR-101 expression was markedly reduced in LSCC cell

A

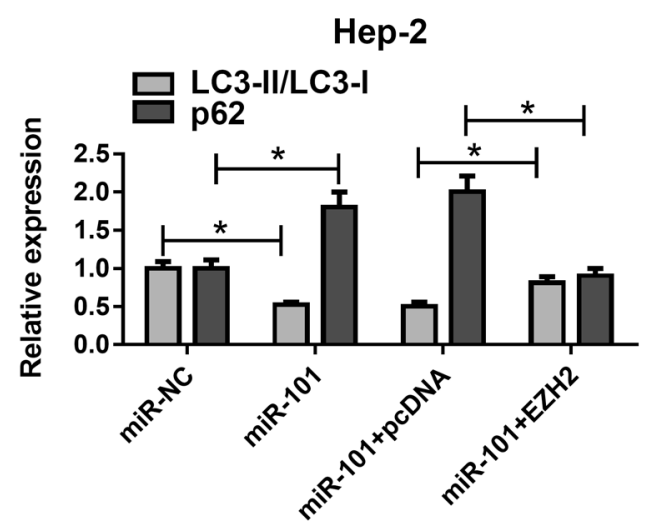

B
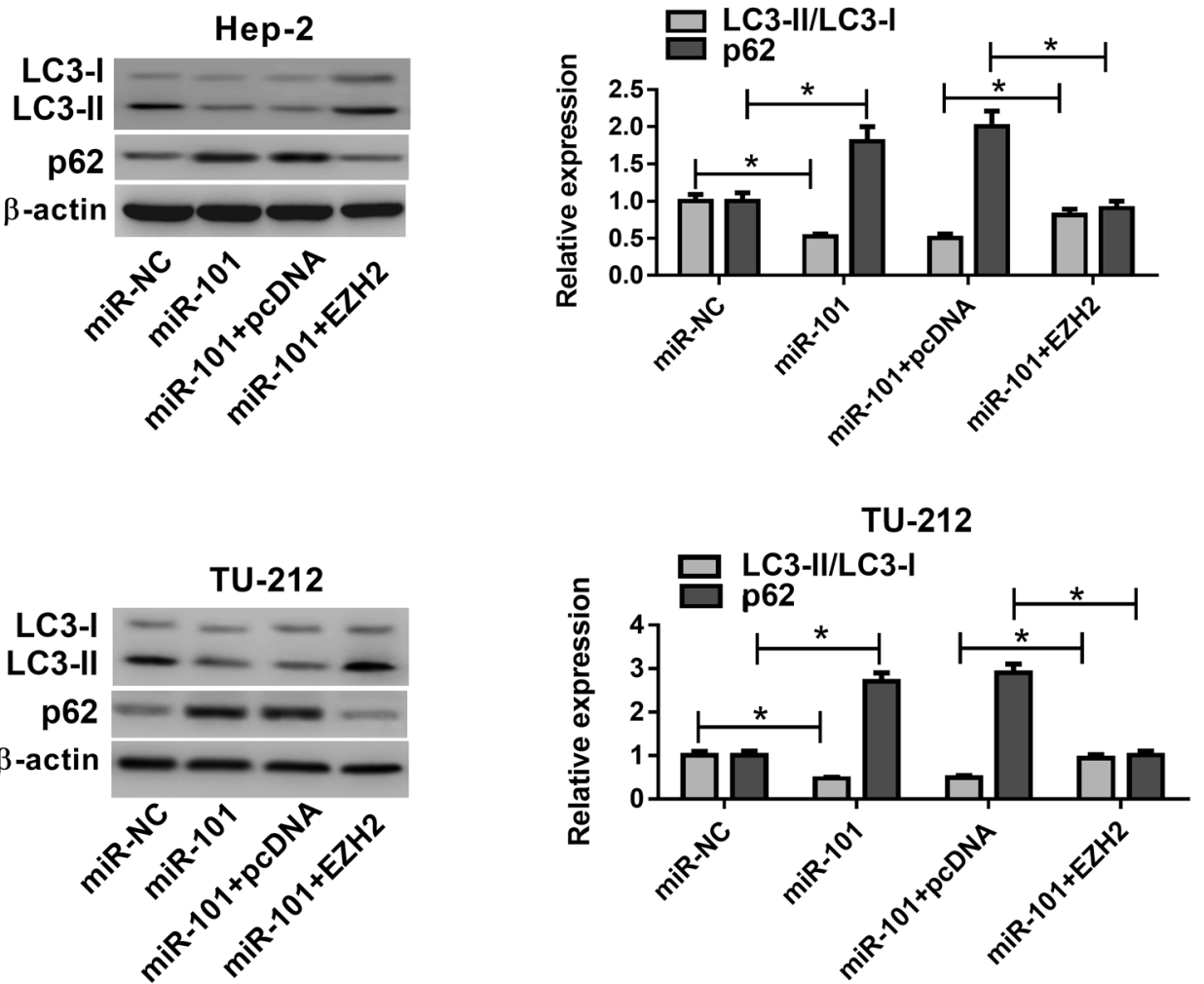

Figure 4. EZH2 up-regulation abrogated the inhibitory effect of miR-101 on autophagy in LSCC cells. At $48 \mathrm{~h}$ after transfection, LC3-II/LC3-I ratio and 162 protein level were examined by western blot assay in Hep-2 (A) and TU-212 (B) cells transfected with si-NC or si-EZH2. (B and C) Hep-2 and TU-212 cells were transfected with miR-NC, miR-101 mimic, miR-101 mimic + pcDNA3.1 vector, or miR-101 mimic + EZH2. Then, the LC3-II/LC3-I ratio and $\mathrm{p} 62$ protein level were measured by western blot assay. ${ }^{*} \mathrm{p}<0.05$. 

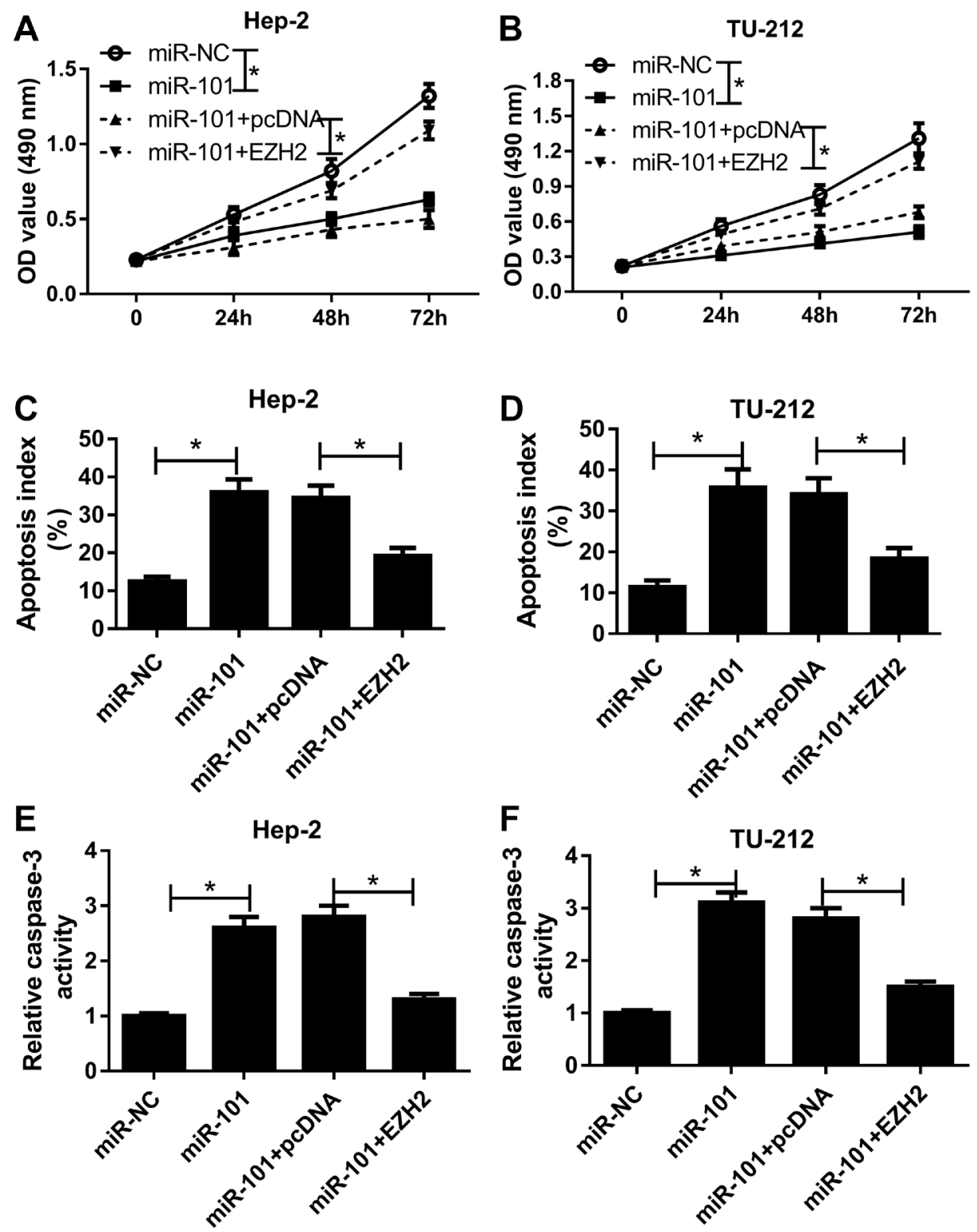

Figure 5. EZH2 restoration weakened miR-101-mediated anti-proliferation and pro-apoptosis effects in LSCC cells. Hep-2 and TU-212 cells were transfected with miR-NC, miR-101 mimic, miR-101 mimic + pcDNA3.1 vector, or miR-101 + EZH2. Next, cell proliferative ability was evaluated by MTS assay at $0,24 \mathrm{~h}, 48 \mathrm{~h}, 72 \mathrm{~h}$ post transfection ( $\mathrm{A}$ and $\mathrm{B})$, cell apoptotic index $(\mathrm{C}$ and $\mathrm{D})$ and caspase-3 activity $(\mathrm{E}$ and $\mathrm{F})$ were determined at $48 \mathrm{~h}$ following transfection. ${ }^{*} \mathbf{p}<0.05$.

lines, which was in line with the results in LSCC tissues in a prior finding [18]. Functional analyses revealed that miR-101 overexpression inhibited autophagy and proliferation and promoted apoptosis in LSCC cells. Moreover, our study revealed that miR-101 overexpression had no much influence on cell proliferation, apoptosis and autophagy in NHOK cells (Figure S1), suggesting the vital values of miR-101 in the therapy of LSCC. However, the effect of miR-101 on autophagy was controversial in different carcinomas. Some studies indicated that miR-101 exerted its anti-tumor effects by inhibiting autophagy in carcinomas such as hepatocellular carcinoma [29] and breast carcinoma [30]. Conversely, Wang et al. showed that ectopic expression of miR-101 facilitated autophagy through down-regulating EZH2 expression in endometrial carcinoma cells [31].

Moreover, we further demonstrated that miR-101 could directly inhibit EZH2 expression in LSCC cells by bioinformatics analysis, luciferase reporter assay and RIP assay. Similar with our results, previous studies also showed that EZH2 was a downstream target of miR-101 and miR-101 could exert its anti-tumor effects by down-regulating EZH2 expression in multiple carcinomas such as hepatocellular 
carcinoma [32], lung squamous carcinoma [33], and embryonal rhabdomyosarcoma [34]. Moreover, our study revealed that EZH2 expression was remarkably upregulated and EZH2 up-regulation abrogated miR-101-mediated anti-autophagy, anti-proliferation and pro-apoptosis effect in LSCC cells. Consistently, EZH2 has been reported to be highly expressed in LSCC tissues and EZH2 overexpression facilitated the tumorigenesis of LSCC [19-21].

Epigenetic mechanisms such as DNA methylation can control miRNA expression, and miRNAs also can regulate the expression of some epigenetic modulators such as polycomb group genes and DNA methyltransferases (DNMTs) in the development of carcinomas [11, 12]. $\mathrm{EZH} 2$, a core component of polycomb repressive complex 2 (PRC2) complex, is a mammalian histone methyltransferase that can silence gene expression via inducing the methylation of lysine 27 on histone 3 (H3K27me) [35]. EZH2 has been reported to be dysregulated at genetic, transcriptional, and posttranscriptional levels in carcinomas [36]. Moreover, EZH2 has been well documented as an oncogenic factor in multiple carcinomas, while some studies indicated that EZH2 functioned as a tumor suppressor in some carcinomas [37, 38]. For instance, the down-regulation of EZH2 resulted in the reduction of cell proliferative and migratory abilities and the increase of autophagic activity and apoptotic rate in colorectal carcinoma cells [39]. However, Mallen-St. Clair et al. pointed out that the depletion of $\mathrm{EZH} 2$ inhibited pancreatic regeneration and promoted $\mathrm{K}-\mathrm{Ras}$ (G12D)-driven neoplastic progression in pancreas in vivo [40].

Taken together, our data showed that miR-101 inhibited autophagy and proliferation and induced apoptosis by targeting EZH2 in LSCC cells, further elucidating the roles and molecular basis of miR-101 in the progression of LSCC and providing some candidate targets for the therapy of LSCC. Moreover, previous studies pointed out that miR-101 might inhibit autophagy by hindering autophagosome formation and fusion of autophagosome and lysosomes $[30,32,41]$. Hence, it is necessary to further investigate the regulatory roles of miR-101/EZH2 axis in the autophagic processes including autophagosome formation, fusion of autophagosome and lysosomes, and autophagosome degradation. Also, it is requisite to explore whether miR-101 could regulate the autophagic activity under stress conditions, such as energy stress or amino acid depletion. Moreover, the mechanisms that EZH2 could regulate autophagy need to be further tested.

Supplementary information is available in the online version of the paper.

Acknowledgements: This research was funded by the key scientific and technological project of Henan province, study has been made on the correlation study of microRNA and laryngeal carcinoma (No. 132102310521).

\section{References}

[1] LYDIATT WM, PATEL SG, O'SULLIVAN B, BRANDWEIN MS, RIDGE JA et al. Head and neck cancers-major changes in the American Joint Committee on cancer eighth edition cancer staging manual. CA Cancer J Clin 2017; 67: 122-137. https://doi.org/10.3322/caac.21389

[2] TAMAKI A, MILES BA, LANGO M, KOWALSKI L, ZENDER CA. AHNS Series: Do you know your guidelines? Review of current knowledge on laryngeal cancer. Head Neck 2018; 40: 170-181. https://doi.org/10.1002/hed.24862

[3] SIEGEL RL, MILLER KD, JEMAL A. Cancer statistics, 2018. CA Cancer J Clin 2018; 68: 7-30. https://doi.org/10.3322/ caac. 21442

[4] BIRKELAND AC, BEESLEY L, BELLILE E, ROSKO AJ, HOESLI $R$ et al. Predictors of survival after total laryngectomy for recurrent/persistent laryngeal squamous cell carcinoma. Head Neck 2017; 39: 2512-2518. https://doi. org/10.1002/hed.24918

[5] TOMEH C, HOLSINGER FC. Laryngeal cancer. Curr Opin Otolaryngol Head Neck Surg 2014; 22: 147-153. https://doi. org/10.1097/MOO.0000000000000032

[6] SCHERL C, MANTSOPOULOS K, SEMRAU S, FIETKAU $\mathrm{R}$, KAPSREITER $\mathrm{M}$ et al. Management of advanced hypopharyngeal and laryngeal cancer with and without cartilage invasion. Auris Nasus Larynx 2016; 44: 333-339. https://doi. org/10.1016/j.anl.2016.08.002

[7] TREIBER T, TREIBER N, MEISTER G. Regulation of microRNA biogenesis and function. Thromb Haemost 2012; 107: 605-610. https://doi.org/10.1160/TH11-12-0836

[8] CHEN Y, FU LL, WEN X, LIU B, HUANG J et al. Oncogenic and tumor suppressive roles of microRNAs in apoptosis and autophagy. Apoptosis 2014; 19: 1177-1189. https://doi. org/10.1007/s10495-014-0999-7

[9] GOZUACIK D, AKKOC Y, OZTURK DG, KOCAK M. Autophagy-Regulating microRNAs and Cancer. Front Oncol 2107; 7: 65. https://doi.org/10.3389/fonc.2017.00065

[10] PENG Y, CROCE CM. The role of MicroRNAs in human cancer. Signal Transduct Target Ther 2016; 1: 15004. https:// doi.org/10.1038/sigtrans.2015.4

[11] RAMASSONE A, PAGOTTO S, VERONESE A, VISONE R. Epigenetics and MicroRNAs in Cancer. International journal of molecular sciences 2018; 19. https://doi.org/10.3390/ ijms 19020459

[12] KUROZUMI A, GOTO Y, OKATO A, SEKI N. DNA Methylation and Dysregulation of miRNA in Cancer, pp 281-296. In: A. Kaneda, Y. Tsukada (Eds.). DNA and Histone Methylation as Cancer Targets. Humana Press 2017, p. 624. ISBN 978-3-319-59784-3

[13] IMAMURA T, KOMATSU S, ICHIKAWA D, MIYAMAE M, OKAJIMA W et al. Low plasma levels of miR-101 are associated with tumor progression in gastric cancer. Oncotarget 2017; 8: 106538-106550. https://doi.org/10.18632/oncotarget. 20860

[14] ZHANG S, WANG M, LI Q, ZHU P. MiR-101 reduces cell proliferation and invasion and enhances apoptosis in endometrial cancer via regulating PI3K/Akt/mTOR. Cancer Biomark 2017; 21: 179-186. https://doi.org/10.3233/CBM-170620 
[15] WANG J, ZENG H, LI H, CHEN T, WANG L et al. MicroRNA-101 Inhibits Growth, Proliferation and Migration and Induces Apoptosis of Breast Cancer Cells by Targeting SexDetermining Region Y-Box 2. Cell Physiol Biochem 2017; 43: 717-732. https://doi.org/10.1159/000481445

[16] LIN C, HUANG F, SHEN G, YIMING A. MicroRNA-101 regulates the viability and invasion of cervical cancer cells. Int J Clin Exp Pathol 2015; 8: 10148-10155.

[17] WANG L, YAO J, SUN H, HE K, TONG D et al. MicroRNA-101 suppresses progression of lung cancer through the PTEN/AKT signaling pathway by targeting DNA methyltransferase 3A. Oncol Lett 2017; 13: 329-338. https://doi. org/10.3892/ol.2016.5423

[18] LI M, TIAN L, REN H, CHEN X, WANG Y et al. MicroRNA-101 is a potential prognostic indicator of laryngeal squamous cell carcinoma and modulates CDK8. J Transl Med 2015; 13: 271. https://doi.org/10.1186/s12967-015-0626-6

[19] HUANG J, ZHOU L, CHEN H, WU C, DUO Z et al. EZH2 is overexpressed in laryngeal squamous cell carcinoma and enhances the stem-like properties of AMC-HN-8 cells. Oncol Lett 2016; 12: 837-846. https://doi.org/10.3892/ol.2016.4704

[20] LUO HN, YUAN J, MA SJ, CHANG HH, YI CX et al. EZH2 promotes invasion and metastasis of laryngeal squamous cells carcinoma via epithelial-mesenchymal transition through H3K27me3. Biochem Biophys Res Commun 2016; 479: 253-259. https://doi.org/10.1016/j.bbrc.2016.09.055

[21] LIAN R, MA H, WU Z, ZHANG G, JIAO L et al. EZH2 promotes cell proliferation by regulating the expression of RUNX3 in laryngeal carcinoma. Mol Cell Biochem 2018; 439: 35-43. https://doi.org/10.1007/s11010-017-3133-7

[22] CHENDRIMADA TP, GREGORY RI, KUMARASWAMY E, NORMAN J, COOCH N et al. TRBP recruits the Dicer complex to Ago2 for microRNA processing and gene silencing. Nature 2005; 436: 740-744. https://doi.org/10.1038/nature 03868

[23] RAMSEY T, GUO E, SVIDER PF, LIN H, SYEDA S et al. Laryngeal cancer: Global socioeconomic trends in disease burden and smoking habits. Laryngoscope 2018; 128: 20392053. https://doi.org/10.1002/lary.27068

[24] AYAZ L, YAROGLU HY, OZCAN C, TAMER L. Differential expression of microRNAs in plasma of patients with laryngeal squamous cell carcinoma: potential early-detection markers for laryngeal squamous cell carcinoma. J Cancer Res Clin Oncol 2013; 139: 1499-1506. https://doi.org/10.1007/ s00432-013-1469-2

[25] LIU Y, LIU J, WANG L, YANG X, LIU X. MicroRNA-195 inhibits cell proliferation, migration and invasion in laryngeal squamous cell carcinoma by targeting ROCK1. Mol Med Rep 2017; 16: 7154-7162. https://doi.org/10.3892/ mmr.2017.7460

[26] GAO W, WU Y, HE X, ZHANG C, ZHU M et al. MicroRNA204-5p inhibits invasion and metastasis of laryngeal squamous cell carcinoma by suppressing forkhead box C1. J Cancer 2017; 8: 2356-2368. https://doi.org/10.7150/jca.19470

[27] HU J, WU C, ZHAO X, LIU C. The prognostic value of decreased miR-101 in various cancers: a meta-analysis of 12 studies. Onco Targets Ther 2017; 10: 3709-3718. https://doi. org/10.2147/OTT.S141652
[28] MA X, BAI J, XIE G, LIU Y, SHUAI X et al. Prognostic significance of microRNA-101 in solid tumor: A meta-analysis. PLoS One 2017; 12: e0180173. https://doi.org/10.1371/journal.pone. 0180173

[29] XU Y, AN Y, WANG Y, ZHANG C, ZHANG H et al. miR-101 inhibits autophagy and enhances cisplatin-induced apoptosis in hepatocellular carcinoma cells. Oncol Rep 2013; 29: 2019-2024. https://doi.org/10.3892/or.2013.2338

[30] FRANKEL LB, WEN J, LEES M, HOYER-HANSEN M, FARKAS T et al. microRNA-101 is a potent inhibitor of autophagy. EMBO J 2011; 30: 4628-4641. https://doi.org/10.1038/ emboj.2011.331

[31] WANG C, LIU B. miR-101-3p induces autophagy in endometrial carcinoma cells by targeting EZH2. Arch Gynecol Obstet 2018; 297: 1539-1548. https://doi.org/10.1007/ s00404-018-4768-7

[32] XU L, BECKEBAUM S, IACOB S, WU G, KAISER GM et al. MicroRNA-101 inhibits human hepatocellular carcinoma progression through EZH2 downregulation and increased cytostatic drug sensitivity. J Hepatol 2014; 60: 590-598. https://doi.org/10.1016/j.jhep.2013.10.028

[33] HOU Y, LI L, JU Y, LU Y, CHANG L et al. MiR-101-3p Regulates the Viability of Lung Squamous Carcinoma Cells via Targeting EZH2. J Cell Biochem 2017; 118: 3142-3149. https://doi.org/10.1002/jcb.25836

[34] VELLA S, POMELLA S, LEONCINI PP, COLLETTI M, CONTI B et al. MicroRNA-101 is repressed by EZH2 and its restoration inhibits tumorigenic features in embryonal rhabdomyosarcoma. Clin Epigenetics 2015; 7: 82. https:// doi.org/10.1186/s13148-015-0107-z

[35] ITALIANO A. Role of the EZH2 histone methyltransferase as a therapeutic target in cancer. Pharmacol Ther 2016; 165: 26-31. https://doi.org/10.1016/j.pharmthera.2016.05.003

[36] YAMAGISHI M, UCHIMARU K. Targeting EZH2 in cancer therapy. Curr Opin Oncol 2017; 29: 375-381. https://doi. org/10.1097/CCO.0000000000000390

[37] YAN KS, LIN CY, LIAO TW, PENG CM, LEE SC et al. EZH2 in Cancer Progression and Potential Application in Cancer Therapy: A Friend or Foe? Int J Mol Sci 2017; 18. https://doi. org/10.3390/ijms18061172

[38] GAN L, YANG Y, LI Q, FENG Y, LIU T et al. Epigenetic regulation of cancer progression by EZH2: from biological insights to therapeutic potential. Biomark Res 2018; 6: 10. https://doi.org/10.1186/s40364-018-0122-2

[39] YAO Y, HU H, YANG Y, ZHOU G, SHANG Z et al. Downregulation of Enhancer of Zeste Homolog 2 (EZH2) is essential for the Induction of Autophagy and Apoptosis in Colorectal Cancer Cells. Genes (Basel) 2016; 7. https://doi. org/10.3390/genes7100083

[40] MALLEN-ST CLAIR J, SOYDANER-AZELOGLU R, LEE KE, TAYLOR L, LIVANOS A et al. EZH2 couples pancreatic regeneration to neoplastic progression. Genes Dev 2012; 26: 439-444. https://doi.org/10.1101/gad.181800.111

[41] RAVIKUMAR B, IMARISIO S, SARKAR S, O'KANE CJ, RUBINSZTEIN DC. Rab5 modulates aggregation and toxicity of mutant huntingtin through macroautophagy in cell and fly models of Huntington disease. J Cell Sci 2008; 121: 1649-1660. https://doi.org/10.1242/jcs.025726 